\title{
Numerical Reconstruction of Residual Stress Fields from Limited Measurements
}

\author{
H. E. Coules ${ }^{1, a^{*}}$, D. J. Smith ${ }^{1, b}$ and K. H. A. Serasli ${ }^{1, c}$ \\ ${ }^{1}$ Department of Mechanical Engineering, University of Bristol, Bristol, BS8 1TR, UK \\ aharry.coules@bristol.ac.uk, bdavid.smith@bristol.ac.uk, 'karim.serasli@bristol.ac.uk
}

Keywords: stress field reconstruction, eigenstrain, finite element analysis.

\begin{abstract}
By finding stress states which are consistent both with any existing experimental measurements and with elasticity theory, residual stress fields can often be reconstructed from incomplete measurement data. We discuss such methods of residual stress reconstruction, their implementation using finite element analysis, and the measurement strategies which enable them.

In general, reconstruction of residual stress fields must be formulated as an inverse problem, which can usually be solved using stress basis functions. However, prior knowledge of the form of the residual stress field and/or underlying eigenstrain distribution often allows the problem to be reduced such that inverse methods become unnecessary, greatly simplifying the analysis. Two examples of when residual stress field reconstruction can be simplified in this way are given.
\end{abstract}

\section{Introduction}

Regardless of the measurement technique applied, residual stress fields are notoriously difficult to determine due to their self-equilibrating nature [1]. Often, there is a fundamental discrepancy between the requirement for accurate residual stress data, and the ability of measurement specialists to acquire it at reasonable cost. For example, although the presence of residual stress in a component or structure is considered in structural integrity assessment standards such as BS7910 [2] and R6 [3], the residual stress distribution is rarely measured in practice due to the difficulty and cost involved. Instead, the residual stress distribution is most often taken from conservative handbook values or from modelling results. At the same time, the majority of practical residual stress measurement methods are quite limited in terms of stress resolution, spatial resolution, and number of stress tensor components measured. For instance, in the deep hole drilling method [4], [5], three in-plane stress components are measured at points along a single line (the hole axis). While this provides a significant amount of stress information, it does not allow the complete residual stress field in an object to be measured explicitly.

Reconstruction of residual stress fields, i.e. inferring the complete stress field from a limited number of measurements, can be carried out by finding distributions of residual stress which are consistent both with existing measurements and with elasticity theory. Generally, stress field reconstruction must be formulated as an inverse problem [6]: basis stress distributions are generated which satisfy equilibrium and boundary conditions, and a linear combination of basis distributions is determined which corresponds closely to the existing measurements. Stress basis functions which satisfy equilibrium and boundary conditions can be generated in a number of ways. These are discussed below. Later, we discuss situations in which an inverse analysis can be avoided.

Eigenstrain-Based Methods. Fundamentally, residual stresses arise due to the presence of incompatible inelastic strains in an object. This can be represented in Cartesian coordinates by a set of modified compatibility equations given by [7]:

$$
\begin{aligned}
& \frac{\partial^{2} e_{x x}}{\partial y^{2}}+\frac{\partial^{2} e_{y y}}{\partial x^{2}}-2 \frac{\partial^{2} e_{x y}}{\partial x \partial y}=-\frac{\partial^{2} \varepsilon_{x x}^{*}}{\partial y^{2}}-\frac{\partial^{2} \varepsilon_{y y}^{*}}{\partial x^{2}}+2 \frac{\partial^{2} \varepsilon_{x y}^{*}}{\partial x \partial y} \\
& \frac{\partial^{2} e_{y y}}{\partial z^{2}}+\frac{\partial^{2} e_{z z}}{\partial y^{2}}-2 \frac{\partial^{2} e_{y z}}{\partial y \partial z}=-\frac{\partial^{2} \varepsilon_{y y}^{*}}{\partial z^{2}}-\frac{\partial^{2} \varepsilon_{z z}^{*}}{\partial y^{2}}+2 \frac{\partial^{2} \varepsilon_{y z}^{*}}{\partial y \partial z} \\
& \frac{\partial^{2} e_{z z}}{\partial x^{2}}+\frac{\partial^{2} e_{x x}}{\partial z^{2}}-2 \frac{\partial^{2} e_{z x}}{\partial z \partial x}=-\frac{\partial^{2} \varepsilon_{z z}^{*}}{\partial x^{2}}-\frac{\partial^{2} \varepsilon_{x x}^{*}}{\partial z^{2}}+2 \frac{\partial^{2} \varepsilon_{z x}^{*}}{\partial z \partial x} \\
& \frac{\partial^{2} e_{x x}}{\partial y \partial z}+\frac{\partial}{\partial x}\left(-\frac{\partial e_{y z}}{\partial x}+\frac{\partial e_{z x}}{\partial y}+\frac{\partial e_{x y}}{\partial z}\right)=-\frac{\partial^{2} \varepsilon_{x x}^{*}}{\partial y \partial z}-\frac{\partial}{\partial x}\left(-\frac{\partial \varepsilon_{y z}^{*}}{\partial x}+\frac{\partial \varepsilon_{z x}^{*}}{\partial y}+\frac{\partial \varepsilon_{x y}^{*}}{\partial z}\right)
\end{aligned}
$$




$$
\begin{aligned}
& \frac{\partial^{2} e_{y y}}{\partial z \partial x}+\frac{\partial}{\partial y}\left(\frac{\partial e_{y z}}{\partial x}-\frac{\partial e_{z x}}{\partial y}+\frac{\partial e_{x y}}{\partial z}\right)=-\frac{\partial^{2} \varepsilon_{y y}^{*}}{\partial z \partial x}-\frac{\partial}{\partial y}\left(\frac{\partial \varepsilon_{y z}^{*}}{\partial x}-\frac{\partial \varepsilon_{z x}^{*}}{\partial y}+\frac{\partial \varepsilon_{x y}^{*}}{\partial z}\right) \\
& \frac{\partial^{2} e_{z z}}{\partial x \partial y}+\frac{\partial}{\partial z}\left(\frac{\partial e_{y z}}{\partial x}+\frac{\partial e_{z x}}{\partial y}-\frac{\partial e_{x y}}{\partial z}\right)=-\frac{\partial^{2} \varepsilon_{z z}^{*}}{\partial x \partial y}-\frac{\partial}{\partial z}\left(\frac{\partial \varepsilon_{y z}^{*}}{\partial x}+\frac{\partial \varepsilon_{z x}^{*}}{\partial y}-\frac{\partial \varepsilon_{x y}^{*}}{\partial z}\right)
\end{aligned}
$$

where $e_{x x}, e_{y y}$ etc. are components of the elastic strain tensor, and $\varepsilon_{x x}^{*}, \varepsilon_{y y}^{*}$ etc. are components for the eigenstrain tensor. One way to generate a set of residual stress bases is to first define a corresponding complete set of eigenstrain bases. Basis distributions of eigenstrain which have been used for this purpose include orthonormal polynomials [8-10] and piecewise linear functions [11], [12]. Finite element analysis is then used to find the distribution of stress in the object which results from each of the eigenstrain bases. The main advantage of eigenstrain-based methods is that a complete set of eigenstrain bases automatically corresponds to the complete set of residual stress distributions.

Stress Function Methods. An alternative approach is to select a set of stress functions (e.g. Airy stress functions for a 2D case) which satisfy the equilibrium and the object's boundary conditions [13], [14]. In a linear elastic material any linear combination of such stress functions will also satisfy these conditions. Therefore, by assuming that the residual stress distribution can be represented by stress functions which satisfy the boundary conditions, the boundary conditions are automatically imposed on the result.

After residual stress basis functions have been generated, both the stress function based and eigenstrain based methods proceed in a similar fashion (this can be seen in references: [15], [16]). A weighted sum of the stress basis functions is constructed, and (some function of) the difference between any available measured residual stress values and the corresponding values from the weighted sum is minimised. This yields a set of weighting coefficients which define the reconstructed residual stress state in terms of the basis stress fields [7].

Limitations of Inverse Analyses. The inverse methods described above are very powerful, but have some significant practical limitations. For all inverse methods, the number of basis functions required rapidly becomes large as the stress field becomes more complex, and consequently the computational cost increases. Typically, gross simplifications must be made to apply inverse eigenstrain or stress function methods to three-dimensional states of residual stress, for example by assuming plane stress or strain states.

In certain cases it is possible to infer additional information about the residual stress distribution without using inverse methods. Two examples are given below. In the first, the incompatible strain resulting from the welding process is known to have a limited spatial extent. Using the stress field in this incompatible region, the residual stress distribution in the remainder of the component can be reconstructed without explicitly determining the eigenstrain distribution. In the second example, assumptions regarding both the form of the eigenstrain distribution and the symmetry of the component are used to reconstruct the residual stress distribution in a clad plate.

\section{Examples}

NeT TG1 Weld Benchmark. In this example, reconstruction of a complex three-dimensional stress field is demonstrated using synthetic measurement data taken from a finite element model of the NeT TG1 weld benchmark specimen [17]. NeT TG1 is a single-pass bead-on-plate TIG weld in SAE Type 316L austenitic stainless steel. A thermo-mechanical model of the welding process for this specimen was developed and used to generate a 'target' residual stress state representative of that in the real plate. The plate was assumed to be symmetric about the weld line, and only one half was modelled (see Fig. 1).

Reconstruction of the complete target residual stress field was carried out using a sub-set of the available residual stress distribution calculated using the welding process model. This sub-set was defined as the region of the solid model having undergone plastic deformation during the welding 
process. This was the only region of the plate which contained incompatible inelastic strain. Since the rest of the plate underwent purely elastic strain during welding, it contained no incompatible inelastic strain once the process was complete. This reconstruction was carried out to demonstrate that the residual stress field in this plastic zone can be used to characterise the complete residual stress field in the specimen. The plastically-deformed region accounted for approximately $17 \%$ of the total volume of the welded plate.

Reconstruction was carried out via an iterative finite element based procedure described in detail elsewhere [18]. Briefly, in each iteration the residual stress state in the eigenstrain-containing region was imposed on the solid model, equilibrium was established, and the resulting stress field in the rest of the object was determined. As the residual stress distribution in the eigenstrain-containing region was iteratively re-imposed, the stress field outside of the 'measured' region asymptotically approached a stationary value. The results, shown in Fig. 2b, illustrate that there is good agreement between the target and reconstructed residual stress fields.

a.

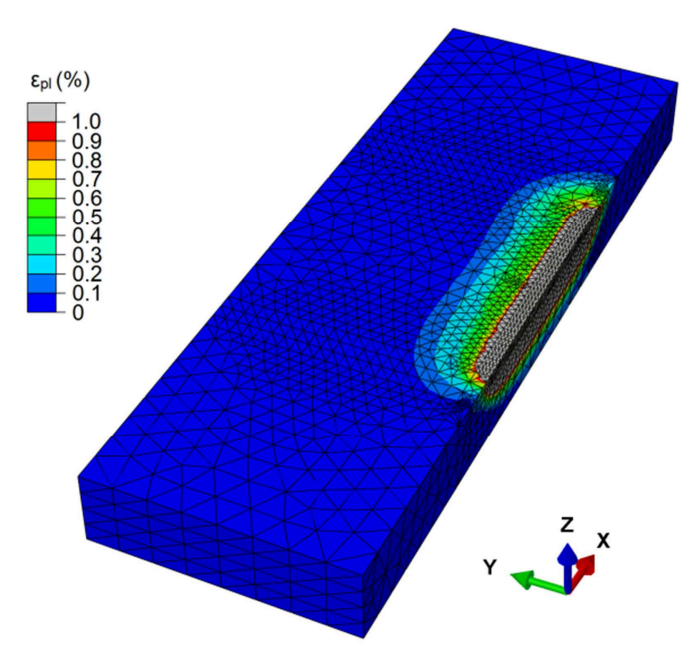

b.

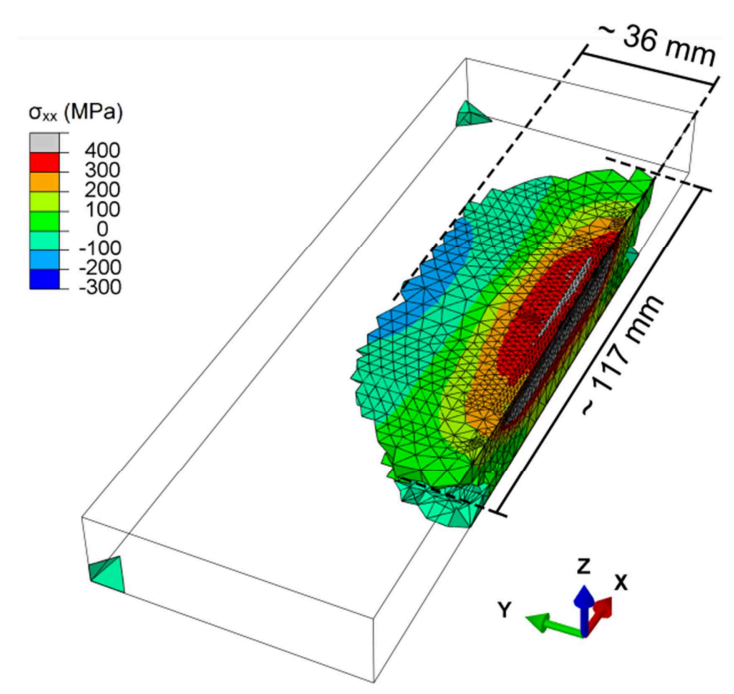

Figure 1: a.) Equivalent plastic strain during welding of NeT TG1 (modelled). b.) Distribution of longitudinal residual stress in the the region of finite plastic strain.

a.

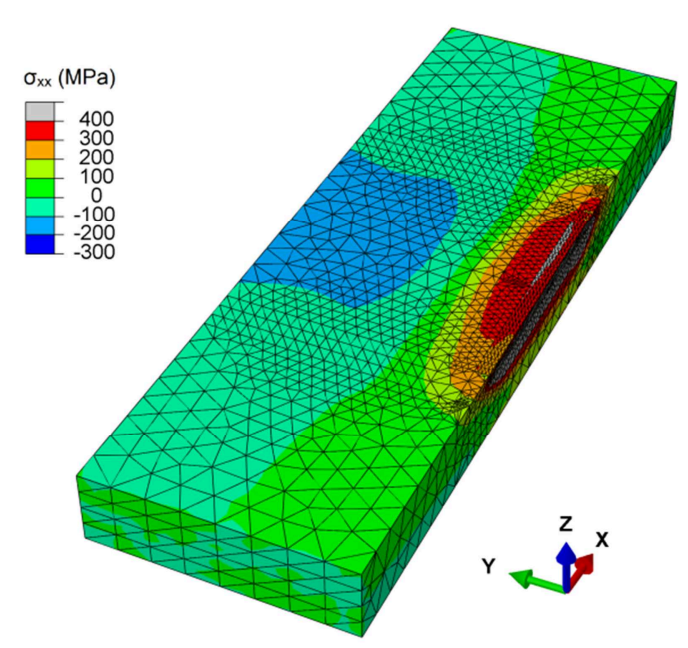

b.

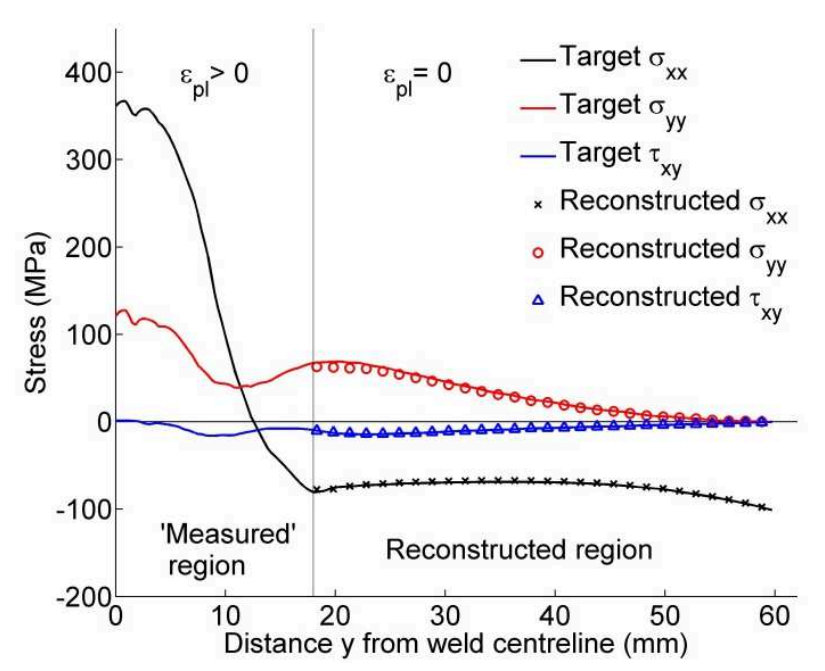

Figure 2: a.) Reconstructed residual stress state (longitudinal), b.) Comparison of target and reconstructed fields along a transverse line. 
In this example, the residual stress field in the eigenstrain-containing region of the plate was assumed to be perfectly characterised. In reality this would not be possible, since all real residual stress measurement methods have practical limitations in terms of spatial and strain resolution. Therefore, in addition to the reconstruction described here, a parametric study of the effects of various types of error and incompleteness in the 'measurement' data was carried out. The effects of limited strain resolution, limited spatial resolution, limited knowledge of size of the eigenstraincontaining region, and a limited number of measured stress tensor components were all investigated. This showed that the iterative reconstruction technique is sufficiently insensitive to errors in the supplied stress data to be used with results from neutron or synchrotron diffraction experiments, and we are currently investigating the application of this method to real measurement results.

TIG-Clad Plate. In this example, the specimen is a rectangular plate of ASME SA 508 Class 3 pressure vessel steel overlaid with SAE Type 308 austenitic stainless steel in multi-layer TIG cladding process. The parent plate prior to cladding had overall dimensions of $300 \mathrm{~mm}$ (length in cladding direction) $\times 150 \mathrm{~mm}$ (width) $\times 50 \mathrm{~mm}$ (thickness). The clad layer, covering most of the upper surface, increased the plate thickness by approximately $8 \mathrm{~mm}$. Since the cladding was uniform over the upper surface of the plate, the eigenstrain distribution was assumed to vary in only one dimension, i.e. through the plate's thickness. At the centre of the plate (half-way along the $\mathrm{x}$ and $\mathrm{y}$ dimensions), symmetry suggests that the elastic strain derivatives in the $\mathrm{x}$ and $\mathrm{y}$ directions are zero. Hence the compatibility equations (Eq. 1) reduce to:

$\frac{\partial^{2} e_{y y}}{\partial z^{2}}=-\frac{\partial^{2} \varepsilon_{y y}^{*}}{\partial z^{2}}$

$\frac{\partial^{2} e_{x x}}{\partial z^{2}}=-\frac{\partial^{2} \varepsilon_{x x}^{*}}{\partial z^{2}}$

$-\frac{\partial^{2} e_{x y}}{\partial z^{2}}=\frac{\partial^{2} \varepsilon_{x y}^{*}}{\partial z^{2}}$

Therefore the eigenstrain distribution was characterised using a single through-thickness incremental Deep Hole Drilling (iDHD) measurement made at the middle of the plate specimen.

iDHD measurements were carried out and the residual elastic strain components $\left(e_{x x}, e_{y y}, e_{x y}\right)$ in the plane of the specimen were obtained, resolved through the depth of the hole (i.e. through the total plate thickness of $58 \mathrm{~mm}$ ). The corresponding eigenstrain components $\varepsilon_{x x}^{*}, \varepsilon_{y y}^{*}$ and $\varepsilon_{x y}^{*}$ were found. This distribution of eigenstrain was then applied as an anisotropic thermal expansion to a solid model of one quarter of the plate and clad layer consisting of approximately 50,000 linear tetrahedral elements. The resulting reconstructed residual stress field is shown in Fig. $3 \mathrm{~b}$.

a.

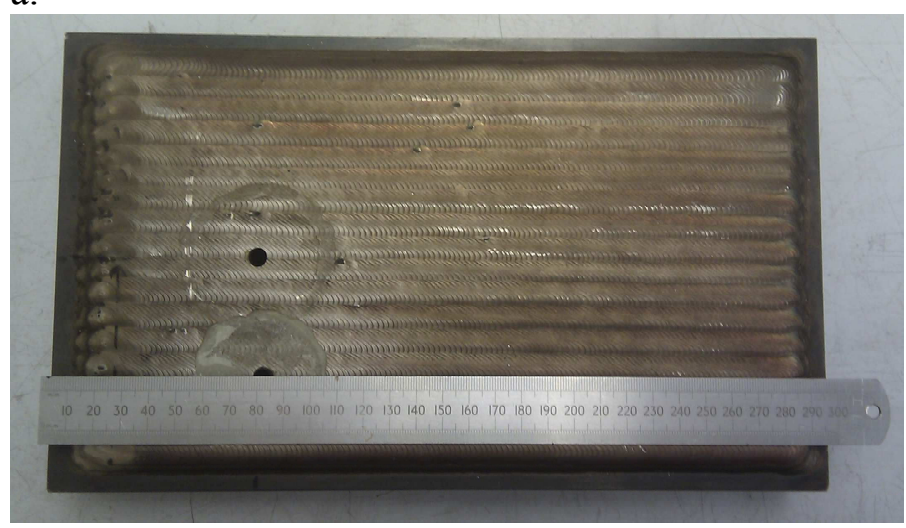

b.

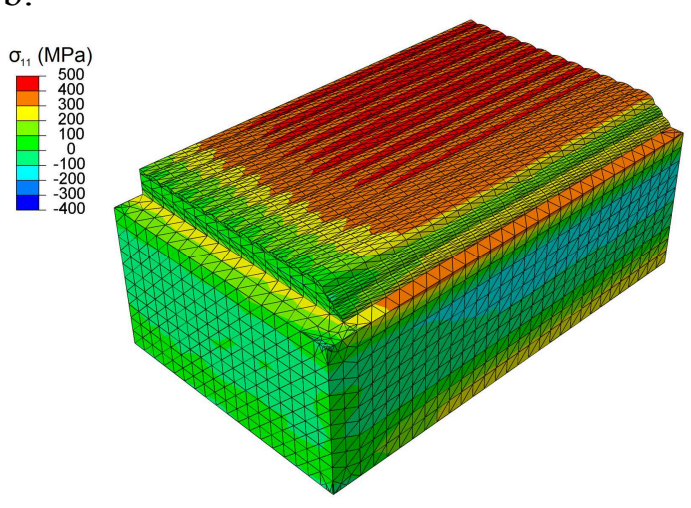

Figure 3: a.) TIG-clad plate, b.) Residual stress field reconstructed from iDHD measurement (longitudinal component shown). 


\section{Concluding Remarks}

Reconstruction of residual stress fields from limited amounts of measurement data can be carried out by using equilibrium and boundary conditions to reduce the number of viable solutions which are compatible with the measured data. This reduction of the solution space is the fundamental principle underlying the application of inverse eigenstrain and stress function methods for residual stress field reconstruction.

Information about the process which introduces the residual stress state, such as the extent of inelastic deformation generated during a peening or welding operation, can be incorporated into this analysis. Very often this allows a problem which would otherwise need to be tackled using inverse methods to be made much more easily solvable. Specifically, when the distribution of incompatible strain can be described using the available measurement data, reconstruction of the rest of the residual stress field can be performed without the use of inverse methods. Therefore, careful selection of measurement methods and locations with the aim of best characterising the underlying eigenstrain distribution can greatly aid residual stress field reconstruction.

\section{Acknowledgements}

Funding for this work was provided under a joint Rolls-Royce/EDF Energy/Royal Academy of Engineering chair awarded to Prof. David Smith. The clad plate measurements were supported by Rolls-Royce plc.

\section{References}

[1] G. S. Schajer and C. O. Ruud, Practical Residual Stress Measurement Methods, 1st ed., G. S. Schajer (Ed.), Wiley, 2013, pp. 1-27.

[2] BSi 7910: Guide to methods for assessing the acceptability of flaws in metallic structures. BSi, 2005.

[3] R6: Assessment of the Inegrity of Structures Containing Defects, Revision 4, British Energy Generation Limited, R/H/R6, 2001.

[4] A. H. Mahmoudi, S. Hossain, C. E. Truman, D. J. Smith, and M. J. Pavier, A new procedure to measure near yield residual stresses using the deep hole drilling technique, Exp. Mech. 49 (2009) 595-604.

[5] D. J. Smith, P. J. Bouchard, and D. George, Measurement and prediction of residual stresses in thick-section steel welds, J. Strain Anal. Eng. 35 (2000) 287-305.

[6] T.-S. Jun and A. M. Korsunsky, Evaluation of residual stresses and strains using the eigenstrain reconstruction method, Int. J. Solids Struct. 47 (2010) 1678-1686.

[7] A. M. Korsunsky, Eigenstrain analysis of residual strains and stresses, J. Strain Anal. Eng. 44 (2009) 29-43.

[8] A. T. DeWald and M. R. Hill, Multi-axial contour method for mapping residual stresses in continuously processed bodies, Exp. Mech. 46 (2006) 473-490.

[9] M. E. Kartal, C. D. M. Liljedahl, S. Gungor, L. Edwards, and M. E. Fitzpatrick, Determination of the profile of the complete residual stress tensor in a VPPA weld using the multi-axial contour method, Acta Mater. 56 (2008) 4417-4428.

[10] A. M. Korsunsky, G. M. Regino, D. Latham, and J. Liu, D. Nowell, and M. Walsh, Eigenstrain analysis of synchrotron X-ray diffraction measurement of residual strains in machined nickel alloy plates, J. Strain Anal. Eng 41 (2006) 381-395. 
[11] T.-S. Jun, A. M. Venter, and A. M. Korsunsky, Inverse eigenstrain analysis of the effect of non-uniform sample shape on the residual stress due to shot peening, Exp. Mech. 51 (2011) $165-174$.

[12] X. Song and A. M. Korsunsky, Fully two-dimensional discrete inverse eigenstrain analysis of residual stresses in a railway rail head, J. Appl. Mech. T. ASME 78 (2011) 031019.

[13] S. A. Faghidian, D. Goudar, G. H. Farrahi, and D. J. Smith, Measurement, analysis and reconstruction of residual stresses, J. Strain Anal. Eng. 47 (2012) 254-264.

[14] G. H. Farrahi, S. A. Faghidian, and D. J. Smith, An inverse approach to determination of residual stresses induced by shot peening in round bars, Int. J. Mech. Sci. 51 (2009) 726-731.

[15] G. H. Farrahi, S. A. Faghidian, and D. J. Smith, An inverse method for reconstruction of the residual stress field in welded plates, J. Press. Vess. T. ASME 132 (2010) 612051.

[16] A. M. Korsunsky, G. M. Regino, and D. A. Nowell, Variational eigenstrain analysis of residual stresses in a welded plate, Int. J. Solids Struct. 44 (2007) 4574-4591.

[17] P. J. Bouchard, The NeT bead-on-plate benchmark for weld residual stress simulation, Int. J. Press. Vess. Pip. 86 (2009) 31-42.

[18] H. E. Coules, D. J. Smith, K. Abburi Venkata, and C. E. Truman, A method for reconstruction of residual stress fields from measurements made in an incompatible region, Int. J. Solids Struct. 51 (2014) 1980-1990. 